On cylindrically converging shock waves shaped by obstacles

V. Eliasson, W. D. Henshaw, D. Appelo

July 18, 2007

Physica D 
This document was prepared as an account of work sponsored by an agency of the United States Government. Neither the United States Government nor the University of California nor any of their employees, makes any warranty, express or implied, or assumes any legal liability or responsibility for the accuracy, completeness, or usefulness of any information, apparatus, product, or process disclosed, or represents that its use would not infringe privately owned rights. Reference herein to any specific commercial product, process, or service by trade name, trademark, manufacturer, or otherwise, does not necessarily constitute or imply its endorsement, recommendation, or favoring by the United States Government or the University of California. The views and opinions of authors expressed herein do not necessarily state or reflect those of the United States Government or the University of California, and shall not be used for advertising or product endorsement purposes. 


\title{
On cylindrically converging shock waves shaped by obstacles
}

\author{
Veronica Eliasson, ${ }^{1,2, *}$ William D. Henshaw ${ }^{3}$ and Daniel Appelö ${ }^{3} \dagger$ \\ ${ }^{1}$ KTH Mechanics, KTH, SE-100 44 Stockholm, Sweden \\ ${ }^{2}$ Department of Mechanical Engineering, University of California, Berkeley, CA 94720, USA \\ ${ }^{3}$ Lawrence Livermore National Laboratory, Livermore, CA 94551 USA
}

\begin{abstract}
Motivated by recent experiments, numerical simulations were performed of cylindrically converging shock waves. The converging shocks impinged upon a set of zero to sixteen regularly space obstacles. For more than two obstacles the resulting diffracted shock fronts formed polygonal shaped patterns near the point of focus. The maximum pressure and temperature as a function of number of obstacles were studied. The self-similar behavior of cylindrical, triangular and square-shaped shocks were also investigated.
\end{abstract}

PACS numbers: 47.10.A-,47.15.ki

\section{INTRODUCTION}

Converging shock waves can be found in a broad range of situations, from astronomical size events like supernovae collapse, to microscopic events such as sonoluminescence when tiny bubbles collapse so strongly as to produce light. Shock waves are an effective method to generate high temperatures and pressures for experimental and engineering purposes and thus remain an area of continued research.

Over the years many experiments have been performed on cylindrically converging shock waves; see e.g. [1, 2]. It is common to use annular shock tubes to create and study converging shock waves. The converging shocks are often visualized by either schlieren photographs or interferograms taken during the focusing process. These methods give a measure of the shock position and shape development as a function of time. With these techniques, it is not possible to measure other quantities, like temperatures and pressures. In a recent paper [3] Eliasson et al. presented experimental results on the light emission occurring at the focal point for converging shock waves of different shapes. By analyzing the response from a photo mulitplier tube, Eliasson et al. found that the amount of emitted light depended on the shape of the converging shock wave. In [3] only a small number of obstacles were considered which resulted in polygons with a few number of sides.

In this paper we present numerical simulations of the experimental setup used in [3]. We consider cylindrically converging shock waves shaped by zero to sixteen obstacles, yielding seventeen different configurations. From monitoring the maximum pressure and temperature as the shocks converge, we find that a low number of obstacles gives a low maximum pressure and temperature, compared to the case with no obstacles. This is consis-

\footnotetext{
*Electronic address: veronica@mech.kth.se

${ }^{\dagger}$ This work was performed under the auspices of the U.S. Department of Energy by University of California, Lawrence Livermore National Laboratory under Contract W-7405-Eng-48.
}

tent with the amount of light observed in [3] for $0,1,3$ and 4 obstacles. However, as we increase the number of obstacles we see a gradual increase in the maximum pressure and temperature; this is somewhat surprising since a greater portion of the initial cylindrical shock is reflected by the obstacles. The present model, the Euler equations for an ideal gas, does not take real gas and ionization effects into account, thus it is not possible to make detailed predictions on light production. Our numerical results suggest that further experiments for more than four obstacles would be of great interest.

Converging shock waves of different polygonal shapes have been studied for example in $[4,5]$. For a polygonal shock the regions of high curvature, such as corners, generally travel faster than the planar parts. This leads to a reconfiguration of the shape of the shock wave during the focusing process. For example, a square-shaped shock wave will transform into an octagon and then back to a square again. This process repeats as the shock focuses provided there are no other disturbances to interrupt it.

In this work, we use the method suggested and tested in experiments by Eliasson et al. [4], to produce converging polygonal shock waves. The numerical simulations were performed using a state of the art adaptive mesh refinement (AMR) flow solver. Disturbances in the form of cylindrical obstacles were introduced in front of an initially cylindrical converging shock. The obstacles used to shape the shock are not small. Therefore it takes some time for the shock front to reach the asymptotic state described by the theory of Schwendeman and Whitham, [5]. From our highly resolved numerical simulations we find that, only at the very final stage of the convergence, a shock perturbed by four obstacles becomes square-shaped. At this stage, the mean radius of the shock is well described by Guderley's, [6], self-similar solution, giving a base solution around which geometrical shock dynamics,[5], can be utilized. The fact that the polygonal shape of the shock is attained only at the final stage, where characteristic length scales (the sides of the polygon) are very small compared to the initial scales (the diameter of initial shock), means that the numerical simulations become quite challenging. 

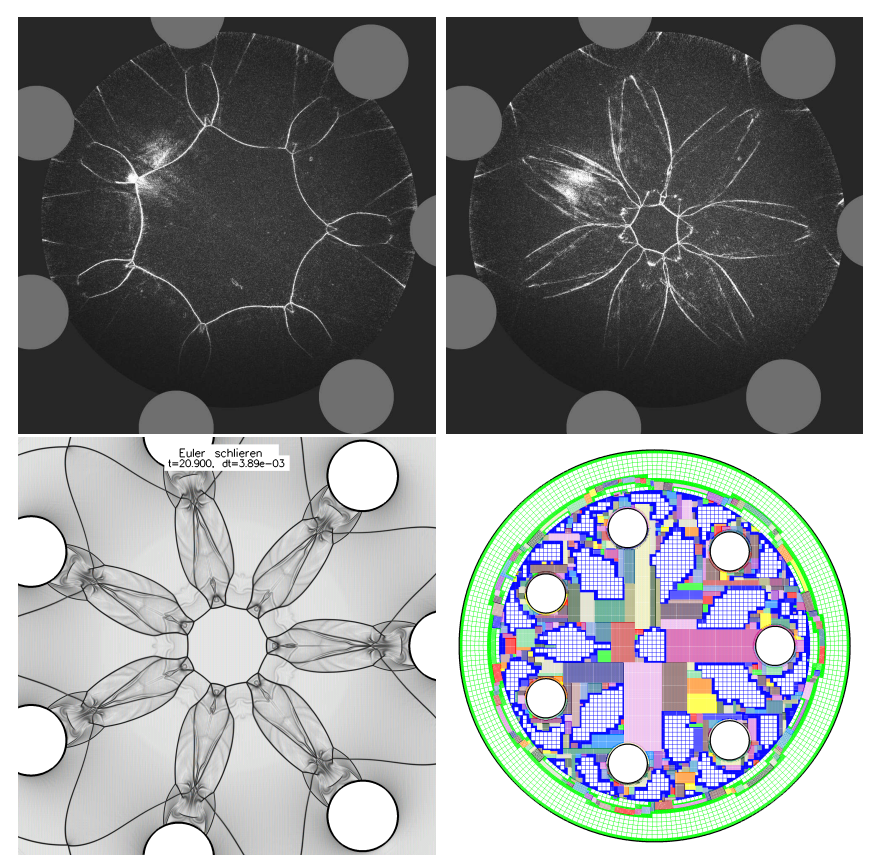

FIG. 1: Experimental and numerical schlieren photographs of a converging polygonal shock wave. Top: experimental results for seven obstacles. Lower left: numerical results. Lower right: An AMR grid with two levels of refinement adapted to the shock structures (every 8th line is plotted).

\section{NUMERICAL METHOD}

The Euler equations of gas dynamics are solved numerically using using a high-order accurate Godunov method, $[7,8]$. The geometry is discretized with overlapping structured grids. Adaptive mesh refinement is used to dynamically track the shocks and contacts. The software, along with references describing the approach can be found at www.llnl.gov/casc/Overture.

\section{NUMERICAL EXPERIMENTS}

The initial conditions in front of the shock are set to be a gas at pressure $p=13.33 \mathrm{kPa}$ (100 torr) and at room temperature $T=294 \mathrm{~K}$, where $\gamma=1.4, R_{g}=287.06$ $\mathrm{J} / \mathrm{KgK}$ and $p=\rho R_{g} T$. The shock front is given an initial shock Mach number of $M=2.4$. The state behind the shock is determined by the standard shock relations. The diameter of the computational domain is set to $150 \mathrm{~mm}$.

The following cases were simulated: an initially cylindrical shock wave perturbed by $0-16$ obstacles (cylinders with a diameter of $15 \mathrm{~mm}$ ) placed in a symmetrical pattern at a radial distance of $46.25 \mathrm{~mm}$ from the focal point, see Figure 1. The boundary conditions on the cylinders are modeled by slip wall conditions. Supersonic outflow boundary conditions are imposed at the perimeter of the computational domain.

In a first set of simulations we compute solutions with
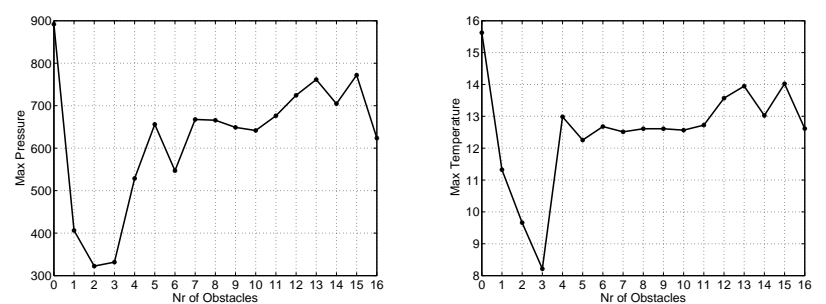

FIG. 2: Maximum pressure and temperature near the focal point as a function of the number of cylinders.

0-16 obstacles to study how quantities like the maximum pressure and temperature vary with the number of obstacles. For these computations the initial grid is composed of a Cartesian background grid (covering most of the domain), an annular perimeter grid and embedded cylindrical grids around each obstacle. The annular grids have a cell size adjusted to the (non-refined) Cartesian grid which has a grid-spacing of $0.2 \mathrm{~mm}$. We use two levels of AMR with a refinement ratio of four yielding a smallest grid size of $50 \mu \mathrm{m}$.

In a second set of experiments, we use an initial grid with a Cartesian grid-spacing of $0.5 \mathrm{~mm}$ but with four levels of AMR with refinement ratio four, yielding a smallest grid size of $7.8125 \mu \mathrm{m}$. With this setup, we limit our simulations to the cases with 0,3 and 4 obstacles and focus on the asymptotic behavior of the converging shocks.

\section{A. Maximum pressure and temperature as a function of the number of cylinders}

The pressure and temperature near the focal point were measured for all seventeen cases. Figure 2 shows the maximum pressure and temperature as a function of the number of cylinders. Figure 7 shows the numerically computed schlieren images for some of these cases. The results show that the undisturbed cylindrical shock gives the highest pressure and temperature near the focal point. This should be expected, since in all other cases, part of the flow is reflected by the obstacles and never reaches the focal point. For a low number of cylinders, $1-6$, the maximum values are low. This is most likely caused by the fact that all parts of the shock front do not reach the focal point at the same time and hence the focusing effect is lost, see Figure 7. Higher pressure and temperatures are obtained for the cases with a larger number of obstacles, 7-13.

\section{B. Comparison to Guderley's self-similar solution}

Guderley, [6], derived a self-similar solution for the radius of the converging shock wave as a function of time, 

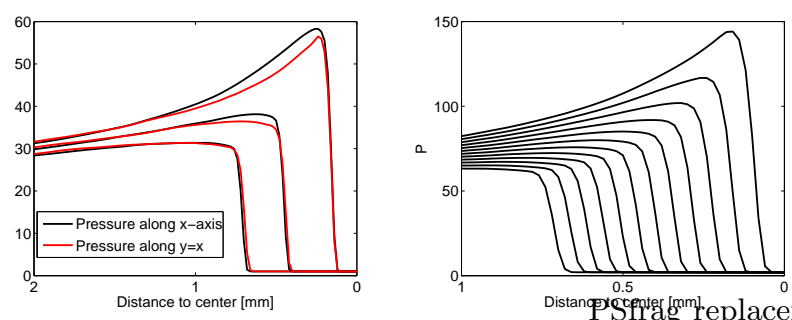

FIG. 3: The case with zero obstacles. Left: the solution along the neg. $x$ axis and the line $y=x, x>0$, at times 22.28, $22.38,22.48$. The difference between the solutions increases as the shock sharpens up. Right: the value of the pressure averaged along the pos. and neg. $x$ and $y$ axes at times $22.34-22.56$ with time spacing 0.02 . Note that the shock is accelerating.

which can be expressed as

$$
R=\xi_{0}\left(t_{c}-t\right)^{\alpha} .
$$

Here $\alpha$ is the self-similar power law exponent, $R$ is the radius of the converging shock wave, $t$ is the time, $t_{c}$ is the time when the shock wave arrives at the center of convergence and $\xi_{0}$ is a constant. Guderley found the selfsimilar power law exponent for cylindrical shock waves to be $\alpha=0.834$ and this has been confirmed by many other investigations, see Table I.

In this study, we investigate when the converging shocks shaped by obstacles are described by Guderely's solution. We fit data from the numerical experiments to equation (1) in order to find the similarity exponent, $\alpha$. We do this for the three cases of a cylinder, a triangle and a square-shaped shock.

Zero Obstacles. To test the accuracy of the numerical algorithms we first consider an unperturbed converging shock and extract the distance between the shock front and the focal point. Starting at time 20 we save solutions every 0.02 time units until time 22.46. For each of the saved solutions we find the position along rays starting at the focal point, where the pressure is half of its global maximum. Precisely, we use rays along the positive and negative $x$ and $y$ axis and the four diagonals in between. We fit the extracted data to equation (1) by minimizing $\sum_{i}\left|R\left(t_{i}\right)-\xi_{0}\left(t_{c}-t_{i}\right)^{\alpha}\right|^{2}$, thus finding $\alpha, t_{c}$ and $\xi_{0}$. Here $R\left(t_{i}\right)$ is taken as the average of the data from the eight rays at time $t_{i}$. The value of the selfsimilar power law exponent, $\alpha=0.844$, agrees well with other values in the literature, see Table I. Note that for the rays used here, the anisotropy in the solution due to grid effects is largest (see Figure 3), thus the errors in the results obtained using these values are likely maximized.

Three Obstacles. The triangular shape was generated by placing three obstacles in an equilateral triangular pattern. Close to the focal point, the shock wave assumes a triangular shape and the similarity exponent can be found. The shock front just before the triangular shape appears is shown in Figure 4 (a)-(b). The
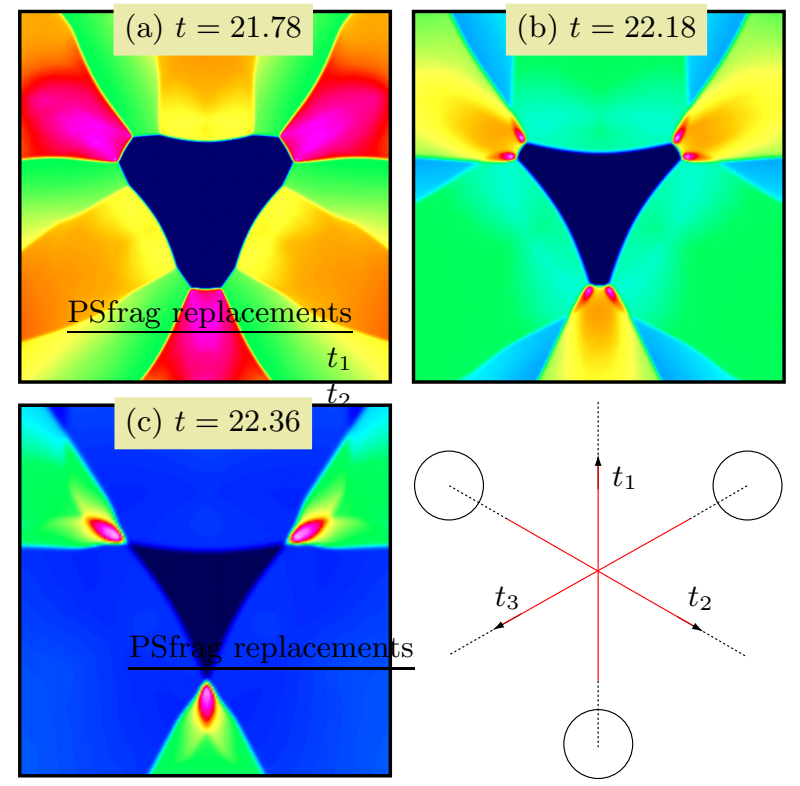

FIG. 4: Contours of the pressure for three obstacles showing the formation of the triangular converging shock.

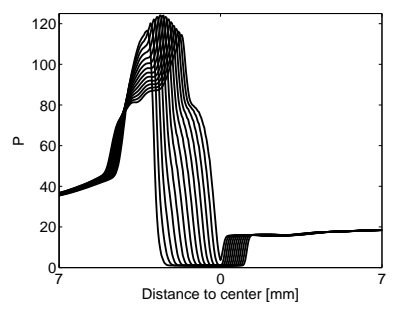

FIG. 5: The value of the pressure with three obstacles averaged along the lines $t_{1}, t_{2}, t_{3}$, of Figure 4 . The solutions are displayed at times $22.28-22.5$ with time spacing 0.02 . The solution to the left of the origin corresponds to the part of the lines $t_{1}, t_{2}, t_{3}$ closest to the obstacles.

plane sides develop as soon as the reflected part of the shock, originating from the reflection off the cylinder, has passed the whole side of the triangle. In Figure 4 (a) the reflected shock is still interacting with the sides of the triangle. In (b), the reflected shocks have passed the sides of the triangle and in (c) a triangle-shaped shock is observed. Once the triangle-shaped shock has formed, it remains for the duration of the focusing process since the plane sides undergo regular reflection, this is consistent with results in [9].

For this experiment the self-similar exponent was computed from solution data along the three lines shown in Figure 4 (d). The pressure, averaged along the three lines, is plotted in Figure 5. Referring to Figure 5, there is a significant difference in the profile of the pressure in the regions to the left and right of the focal point at the origin; we therefore make two fits to the data. Using the averaged values of the solutions at times 22.34 to 22.56 we get a self-similar exponent $\alpha=1.155$ for the data to the left and $\alpha=0.977$ to the data on the right. The 
fact the similarity exponent is not exactly equal to unity probably results from the sides not being perfectly plane until the very last stages of the focusing process (see, Figure $4(\mathrm{c})$ ).

Four Obstacles. A square-shaped shock was obtained by perturbing a cylindrical shock with four obstacles placed in a square formation, see Figure 6 . A square-shaped shock undergoes Mach reflection if the shock Mach number is larger than 1.24, [9], as is the case here. This means that when two plane sides meet in a corner, a new shock (Mach stem) is created. The Mach stem travels faster than the adjacent plane sides and will consume these; repeating for the rest of the focusing process. In the present setup, the Mach stem will form along the lines $s_{2}$ and $s_{4}$ (see Figure $6(\mathrm{~d})$ ) and expand outwards towards the lines $s_{1}$ and $s_{3}$. When adjacent stems meet the square has turned 45 degrees.

Because of this reconfiguration process it is impractical to detect the location of the shock along rays. Instead we compute the area of the domain where the pressure is within $5 \%$ of its quiescent state. Assuming the area to be

\begin{tabular}{ll}
\hline & Self similar exponent \\
\hline Present results (zero obstacles) & 0.844 \\
Present results (four obstacles) & 0.835 \\
Guderley (1942), [6] & 0.834 \\
Butler (1954) & 0.835217 \\
Stanyukovich (1960) & 0.834 \\
Welsh (1967) & 0.835323 \\
Mishkin \& Fujimoto (1978) & 0.828 \\
Nakamura (1983) & $0.8342, M_{s}=4.0$ \\
& $0.8345, M_{s}=10.0$ \\
de Neef \& Nechtman* (1978) & $0.835 \pm 0.003$ \\
Kleine* (1985) & $0.832+0.028,-0.043$ \\
Takayama* (1986) & $0.831 \pm 0.002$ \\
\hline
\end{tabular}

TABLE I: Self similarity exponents for converging cylindrical shock waves. *Experiments.

proportional to the square of the mean radius, we can use the square root of the area instead of $R$ to find $\alpha$ from (1). Using solutions from the final stages, corresponding to times 21.96 to 22.7 (with a time step of 0.02), we obtain a self-similar exponent $\alpha=0.835$. This in agreement with the theory in [5].

It should be noted that in general the computed value of the self-similar exponent depends slightly on the data set used. In particular for the case of four obstacles, there is a tendency for the computed value of $\alpha$ to be somewhat larger when solutions at earlier times are included.

\section{CONCLUSIONS}

The shape of the shock front and the diffraction pattern behind the shock in the numerical simulations agree
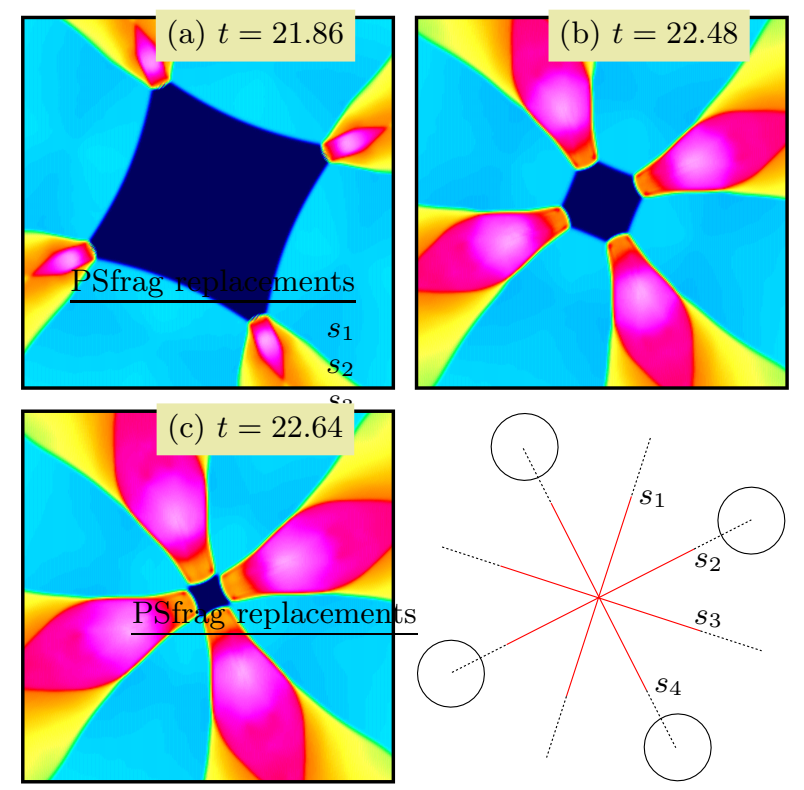

FIG. 6: Contours of the pressure for four obstacles. The square shaped shock front periodically reforms, rotated by 45 degrees.

well with the experimental results in [4]. The maximum pressure and temperature near the focal point were computed using 0-16 cylindrical obstacles. The highest maximum pressure and temperature occurred with zero obstacles. With a small number of obstacles, 1-6, the maximum pressure and temperature were lower than with a large number of obstacles, $7-16$. During the final stages of the focusing process, a self-similar solution is obtained for the triangular and the square-shaped shock. The triangle-shaped shock undergoes regular reflection and the same shape remains during the focusing process. For the triangle, the self-similar exponent depends on the direction in which the location of the shock front is measured. For the two directions measured here, the exponents were $\alpha=0.977$ and $\alpha=1.155$, compared to the expected value of one. The square-shaped shock undergoes Mach reflection and the self-similar exponent was found to be $\alpha=0.835$ in agreement with other published results.

\section{Acknowledgments}

V.E. thank Prof. A.J. Szeri at the Department of Mechanical Engineering, University of California, Berkeley, for hosting her visit and providing computational resources. The work of V.E. was supported by Prof. H.P. Alfredsson at KTH Mechanics, Stiftelsens Bengt Ingeströms Stipendiefond and Helge Ax:son Johnsons Stiftelse. The authors acknowledge valuable discussions with Prof. D.W. Schwendeman. 

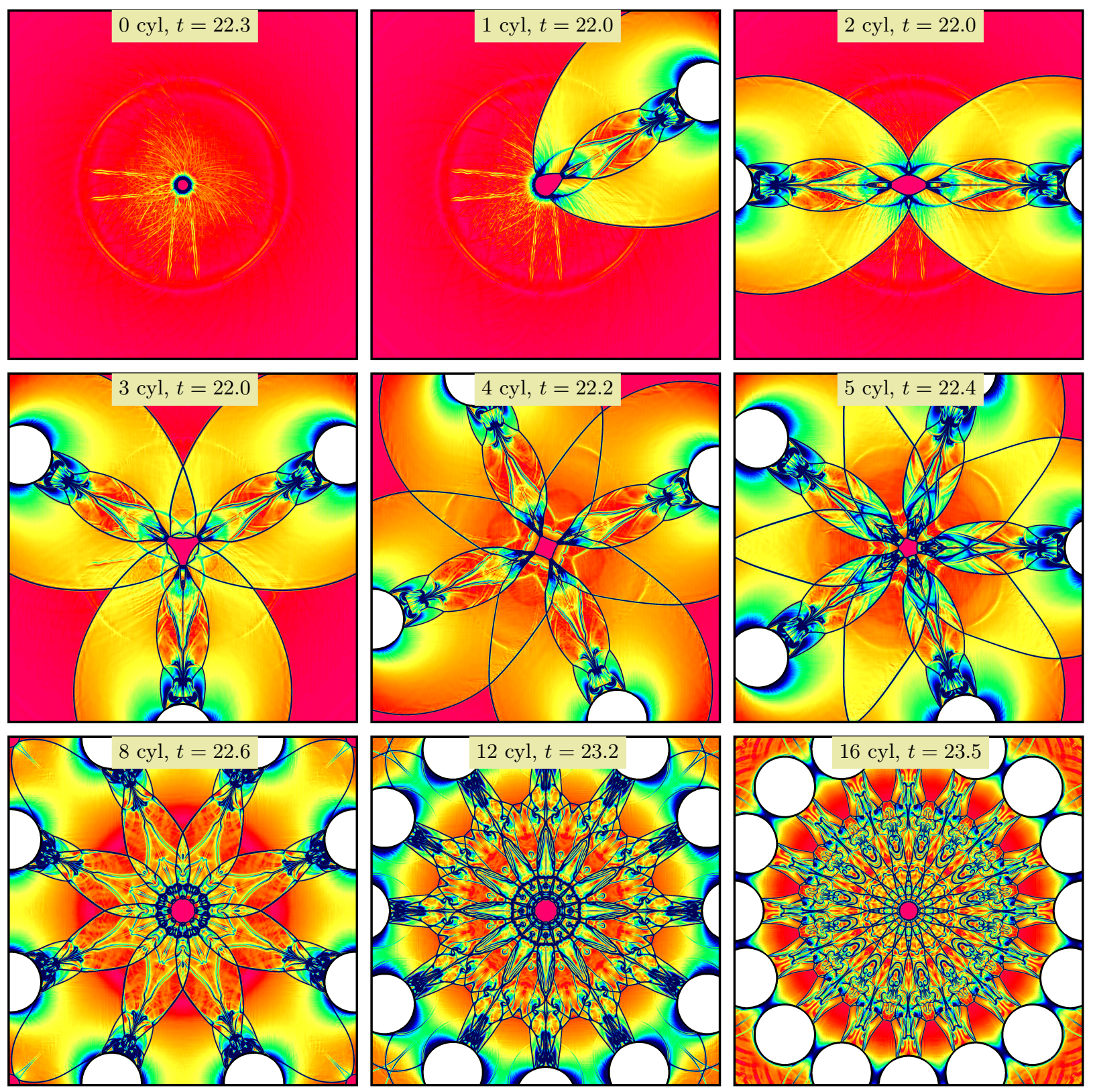

FIG. 7: Numerically computed schlieren images for a converging shock diffracted by $0,1,2,3,4,5,8,12$ and 16 cylindrical obstacles. The dominant portion of the shock is located near the focal point. This part of the shock front is far from circular in cases $1-5$, whereas it is close to circular in cases 8-16.

[1] K. Takayama, H. Kleine, and H. Grönig, Exp. Fluids 5, 315 (1987).

[2] M. Watanabe, O. Onodera, and K. Takayama, Shock Waves@ Marseille IV (1995).

[3] V. Eliasson, N. Apazidis, N. Tillmark, and A. J. Szeri, Submitted to Physics of Fluids (2007).

[4] V. Eliasson, N. Apazidis, N. Tillmark, and M. B. Lesser, Shock waves 15, 205 (2006).

[5] D. W. Schwendeman and G. B. Whitham, Proc. R. Soc.
Lond. A A413, 297 (1987).

[6] G. Guderley, Luftfahrt Forsch 19, 302 (1942).

[7] W. D. Henshaw and D. W. Schwendeman, J. Comput. Phys. 191, 420 (2003).

[8] W. D. Henshaw and D. W. Schwendeman, J. Comput. Phys. 216, 744 (2006), ISSN 0021-9991.

[9] S. I. Betelu and D. G. Aronson, Phys. Rev. Lett. 87, 074501 (2001) 